\title{
Hybrid Stabilizing Control on a Real Mobile Robot
}

\author{
To establish empirical verification of a stabilizing controller for non-holo- \\ nomic systems, the authors implement a hybrid control concept on a \\ 2-DOF mobile robot. Practical issues of velocity control are also \\ addressed through a velocity controller which transforms the mobile \\ robot to a new system with linear and angular velocity inputs. Experi- \\ ments in the physical meaning of different controller components \\ provide insights which result in significant improvements in con- \\ troller performance.
}

$\boldsymbol{S}^{\mathrm{t}}$ tabilization of non-holonomic systems to an arbitrary point in state space is in general quite difficult. An example of a non-holonomic system is a car, which can be steered to any position and any orientation in free space. However, the freedom of motion of a car is limited: it cannot move sideways. Thus complicated maneuvering is needed to bring the car to an arbitrary position and orientation. Possible applications of non-holonomic stabilizing control might be in industrial environments, where wheeled mobile robots have to visit work stations, or in automated vehicle parking.

The kinematics of many practical non-holonomic systems, such as mobile robots, cars, and trucks with one or more trailers, can be modeled as nonlinear systems in chained form $[6,7,11]$ (see also the next section). Because of its practical importance the stabilization of such systems has received much attention in recent literature. Stabilization cannot be achieved by smooth static state feedback $[2,6]$. For this reason other solutions have been proposed, based, for instance, on time-varying feedback [4, $9,10]$, discontinuous feedback [11] and hybrid feedback [3]. In most of these publications, the theoretical correctness of the approaches is demonstrated by computer simulations. However, because such simulation studies usually neglect practically important aspects, such as nonlinear dynamics, rolling friction and compliance of the mechanical structure, their value is limited. Moreover, in our opinion a final justification for these research efforts lies in the practical implementation.
To the best of our knowledge, this paper offers the first reported empirical verification of a stabilizing controller for non-holonomic systems. For this purpose, a hybrid control concept [3] was implemented on a mobile robot with two degrees of freedom ( 2 DOF), which can be described as a onechained nonlinear system (see $[6,11]$ and other recent work on chained systems). This experimental robot system has two driven wheels and a castor to carry the mechanical structure.

Special attention is paid to a number of controller implementation issues. One issue is that the hybrid controller is designed for a system with velocity input ("kinematics"), whereas in practice we have to deal with torque or force input ("dynamics"). Hence, a velocity controller was developed that transforms the mobile robot to a new system with two velocity inputs: linear velocity and angular velocity. The hybrid stabilizing controller is implemented on this velocity-controlled mobile robot. Another issue is the selection of controller parameters. Experiments provided useful insight in the physical meaning of different controller components. This insight allowed us to significantly increase the control performance.

In the next section, the hybrid controller, proposed by Canudas de Wit et al. [3], is applied to a 2-DOF mobile robot. Then the hardware and practical setup of the mobile robot are discussed, followed by a description of the implementation of the hybrid controller on the velocitycontrolled mobile robot. Finally we give the experi- 
mental results and conclusions.

Part of the present paper is published in [8].

\section{HYBRID STABILIZATION OF A MOBILE ROBOT}

The applied controller is based on a general control concept for n-dimensional, nonlinear systems in one-chained form [3]. The kinematics of the mobile robot can be transformed to 3dimensional, one-chained form by means of a coordinate transformation and a feedback transformation. The resulting controller consists of two parts. A discrete-time part that stabilizes the error in the driving direction and a piecewise continuous-time part that stabilizes the lateral error. In order to take into account dynamical limitations of the mechanical structure, the one-chained form, derived from the kinematics of the mobile robot, is extended by two integrators in a special way.

\section{Transformation to Chained Form}

The kinematics of a 2 DOF mobile robot can be described by

$$
\begin{aligned}
& \dot{x}=v \cos (\varphi) \\
& \dot{y}=v \sin (\varphi) \\
& \varphi=\omega
\end{aligned}
$$

Here $x, y$ and $\varphi$ are the position and orientation of the vehicle and $v$ and $\omega$ are the linear and angular velocity of the vehicle. The kinematics, given in (1) can be realized by means of two driven wheels and a castor. The castor is needed for carrying the mechanical structure. It plays no active role in the driving mechanism of the mobile robot. This configuration is shown in Figure 1.

For this type of wheel configuration the point $(x, y)$ corresponds to the midpoint of the rear axis. The orientation of the vehicle is the angle $\varphi$ relative to the $x$-axis of the world coordinate system. The linear velocity $v$ is the velocity in driving direction of the midpoint of the rear axis; the angular velocity $\omega$ is the temporal derivative of the angle $\varphi$.

The kinematics (1) can be transformed locally to onechained form, using the following coordinate transformation, which is intended to be used for values of $\varphi$ in the interval $<-1 / 2 \pi, 1 / 2 \pi>$.

$$
\begin{aligned}
& x_{1}=x \\
& x_{2}=\tan (\varphi) \\
& x_{3}=y
\end{aligned}
$$

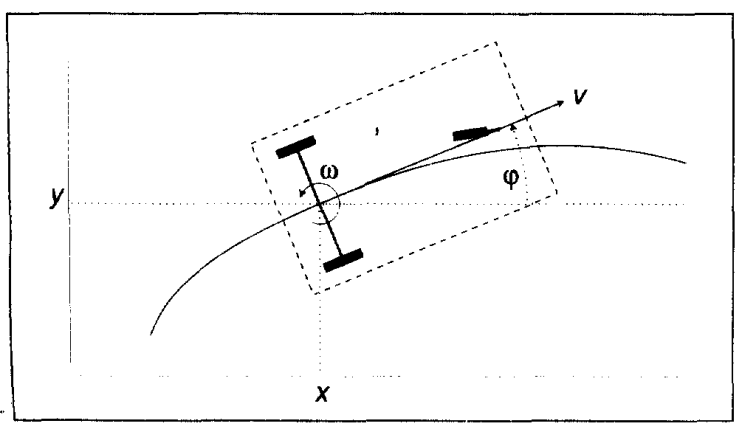

Fig. 1. Wheel configuration of mobile robot

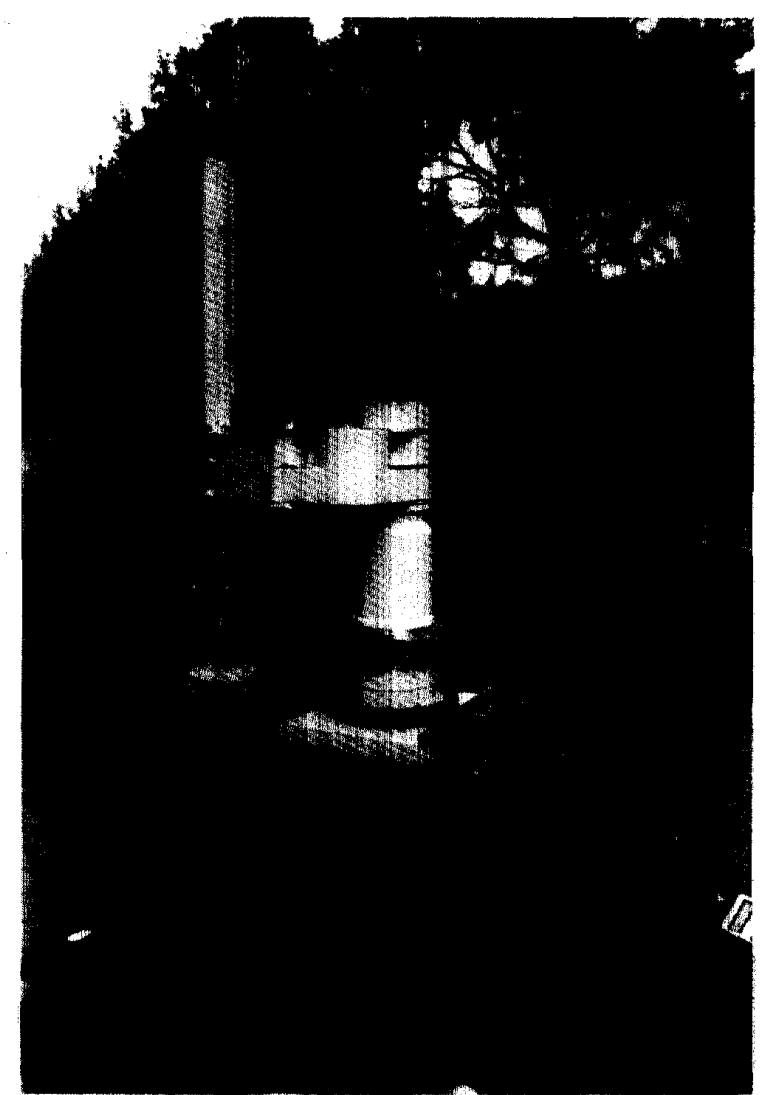

Fig. 2. Picture of MART-robot

The transformation requires the inputs to be defined as

$$
\begin{aligned}
& u_{1}=v \cos (\varphi) \\
& u_{2}=\frac{\omega}{\cos ^{2}(\varphi)}
\end{aligned}
$$

Using (2) and (3), the system transforms into a 3-dimensional system with one chain [3], with states $x_{1}(t), x_{2}(t)$ and $x_{3}(t)$ and inputs $u_{1}(t)$ and $u_{2}(t)$.

$$
\begin{aligned}
& \dot{x}_{1}=u_{1} \\
& \dot{x}_{2}=u_{2} \\
& \dot{x}_{3}=u_{1} x_{2}
\end{aligned}
$$

Subsystem (4a) can be stabilized by applying piece-wise constant input signals $u_{1}(t)$. During one time interval, in which $u_{1}(t)$ is constant, the remaining subsystem $(4 \mathrm{~b}, \mathrm{c})$ can be regarded as a time-invariant linear system with one input signal $u_{2}(t)$. This system is controllable as long as $u_{1}(t) \neq 0$, and in this case a continuous-time controller exists, that stabilizes $(4 \mathrm{~b}, \mathrm{c})$. The continuous time controller has to be designed, such that $x_{2}(t)$ and $x_{3}(t)$ converge (much) faster than $x_{1}(t)$. 
Extension of One-chained System

If a discrete-time controller is used for $x_{1}(t)$, then $u_{1}(t)$, and hence the velocity, has to change abruptly at each new sampling interval. This is not possible for a real mobile robot, because of its inertia. The problem of abruptly changing input velocities can be solved by simply adding integrators in cascade to each of the inputs of the one-chained system. In [3], however, integrators are added in the following particular way:

$$
\begin{aligned}
& u_{1}(t)=\int_{01}^{i} v_{1}(\tau) \mathrm{d} \tau \\
& u_{2}(t)=\int_{i}^{1} v_{1}(\tau) \mathrm{d} \tau \int_{01}^{b_{1}} v_{2}(\tau) \mathrm{d} \tau
\end{aligned}
$$

The reason for adding the integrators in this particular way is that the extended system can be rewritten in chained form again, by introducing two new state variables, $w_{1}(t)$ and $w_{2}(t)$ :

$$
\begin{aligned}
& \dot{w}_{1}(t)=v_{1}(t) \\
& \dot{x}(t)=w_{1}(t) \\
& \dot{w}_{2}(t)=v_{2}(t) \\
& \dot{x}_{2}(t)=w_{1}(t) w_{2}(t) \\
& \dot{x}_{3}(t)=w_{1}(t) x_{2}(t)
\end{aligned}
$$

Here $v_{1}(t)$ and $v_{2}(t)$ are the new inputs of the system. If they are piece-wise continuous in time, then $u_{1}(t)=w_{1}(t)$ and $u_{2}(t)=w_{1}(t) w_{2}(t)$ are continuous in time. Subsystem (6a) again is stabilized using a discrete-time controller and subsystem (6b) is stabilized using continuous-time control.

If $v_{1}$ is piecewise constant during time intervals $\mathrm{I}_{\mathrm{k}}=[k \delta$ $(k+1) \delta$, where $\delta$ is the sample interval of the discrete-time controller, then the discrete-time system

$$
\begin{aligned}
& w_{1}(k+1)=w_{1}(k)+\delta v_{1}(k) \\
& x_{1}(k+1)=x_{1}(k)+\delta w_{1}(k)+\frac{1}{2} \delta^{2} v_{1}(k)
\end{aligned}
$$

is an exact, discrete representation of $(6 a)$. Here $v_{1}(k), w_{1}(k)$ and $x_{1}(k)$ are shorthand notations for $v_{1}, w_{1}$ and $x_{1}$ at time $k \delta$.

\section{Geometric interpretation}

For each of the quantities, introduced above, a geometric interpretation can be given. For $x_{1}$ and $x_{3}$ this is exact. For the other quantities, except $u_{2}$, it is an approximation with an accuracy better than $7 \%$ as long as $|\varphi|<20^{\circ}$.

$$
\begin{aligned}
& x_{1}: x \text {-position of vehicle } \\
& x_{2}: \text { orientation of vehicle } \\
& x_{3}: y \text {-position of vehicle } \\
& u_{1}: \text { velocity in driving direction } \\
& u_{2}: \text { angular velocity (within } 15 \% \text { for }|\varphi|<20^{\circ} \text { ) } \\
& w_{1}: \text { velocity in driving direction } \\
& w_{2}: \text { momentaneous curvature }(\omega / v) \text { of trajectory } \\
& v_{1}: \text { acceleration in driving direction } \\
& v_{2}: \text { time derivative of momentaneous curvature }
\end{aligned}
$$

Stabilization of Extended One-chain System

Here the hybrid controller from [3] is given for the specific case of the transformed kinematics of the mobile robot, extended with the two integrators $(6 \mathrm{a}, \mathrm{b})$.

Consider the subsystems (7) and (6b), together with the following hybrid controller:

$$
\begin{aligned}
v_{1}(k) & =\mathrm{K}(\xi(k))+ \\
& H(\xi(k)) \alpha(\|z(k \delta)\|) \\
v_{2}(t)= & \left.-\mid w_{1}(t)\right)\left(k_{z, 1} w_{2}(t)+\right. \\
& \left.\operatorname{sign}\left(w_{1}(t)\right) k_{z, 2} x_{2}(t)+k_{z, 3} x_{3}(t)\right),
\end{aligned}
$$

where

$$
\begin{aligned}
& z(t)=\left[w_{2}(t) x_{2}(t) x_{3}(t)\right]^{\mathrm{T}} \\
& \xi(k)=\left[w_{1}(k) x_{1}(k)\right]^{\mathrm{T}},
\end{aligned}
$$

and $K(\xi)$ is any asymptotically stabilizing discrete-time feedback controller for subsystem (7) and

$$
\mathrm{H}(\xi(k))=\operatorname{sign}\left(w_{1}(k)+\frac{\delta}{2} \mathrm{~K}(\xi(k))\right)
$$

$\alpha(r)$ is any continuous, strictly increasing function of $r$, with the property $\alpha(r) \rightarrow \infty$ if $r \rightarrow \infty$, and the constants $k_{z, j}(1 \leq j \leq 3)$ are such that all zeros of

$$
\lambda^{3}+k_{*, 1} \lambda^{2}+k_{z, 2} \lambda+k_{z, 3}
$$

have negative real part. Then for any positive error bound $\varepsilon$, parameters $k_{z, j}(1 \leq j \leq 3)$ and $\delta$, and functions $\alpha($.$) and \mathrm{K}($.$) can$ be found, such that for any given initial state of system $(6 b, 7)$ the system with the feedback $(8 a, b)$ converges within a ball with radius $\varepsilon$ around zero.

In the practical implementation of this controller the parameters $k_{z j}(1 \leq j \leq 3)$ and the functions $\alpha($.$) and K($.$) must be tuned$ in order to achieve an acceptable performance. This subject was covered in our discussion of the implementation of the hybrid controller. The norm $\|z(k)\|$ may be any norm, but Canudas de Wit et al. [3] use a Euclidian norm for their simulation study.

\section{MOBILE AUTONOMOUS ROBOT TWENTE}

The MART project results from a preliminary study by Abrahams [1], who developed a concept for a future automated assembly hall. Instead of assembly of products on a conveyor belt, a more flexible and more robust system is proposed, in which a set of mobile robots takes care of the assembly tasks. In this concept, each mobile robot consists of a vehicle which carries a manipulator. Assembly can be done on the vehicle (also during motion for increased throughput) and on fixed work stations. The assembly concept allows the use of 2 DOF mobile robots, as long as they can be positioned sufficiently 


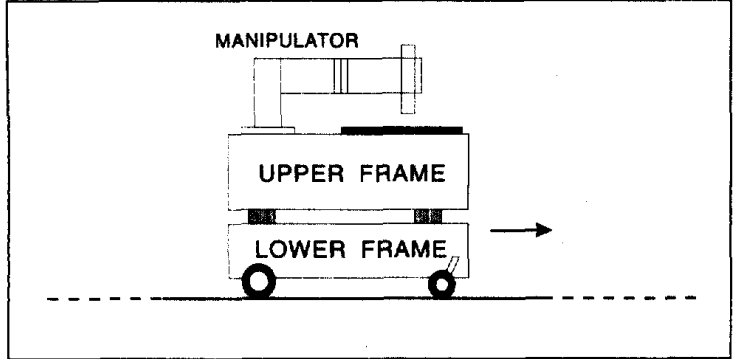

Fig. 3. Schematic drawing of final MART-robot

accurately. We will now present a fairly detailed overview of the physical properties of the mobile robot.

Mechanical Structure of the Mobile Robot

The mobile robot, as it is used for the experiments described below, is to be used in a coarse industrial environment with floor irregularities, dust and varying temperatures. In Figure 2 a picture of the mobile robot in its current state is given. During the experiments the manipulator was not yet mounted on the mobile robot, but for the experiments with the hybrid controller this is of no importance.

The driving system of the mobile robot, which has to move from fixed work station to fixed work station, should be robust and inexpensive. Because of its ruggedness and simplicity a driving system was chosen with two driven wheels and one castor (see Figure 1). In the intended application the mobile robot only needs to move forward in normal operation, and the castor is not expected to cause problems in this application.

Because the floor of the factory hall may be contaminated with many irregularities like thresholds and little stones, the manipulator needs to be suspended. The vehicle consists of upper and lower parts, which are connected to each other by means of soft springs. The lower part contains the wheels and drive motors, while the upper part of the vehicle carries the manipulator, the batteries and the necessary electronics, and the soft springs provide a good suspension, without the need for very soft tires. Figure 3 shows a schematic drawing of the mobile robot.

In the experimental setup the lower part of the vehicle has a mass of $75 \mathrm{~kg}$. The upper part of the vehicle has a mass of

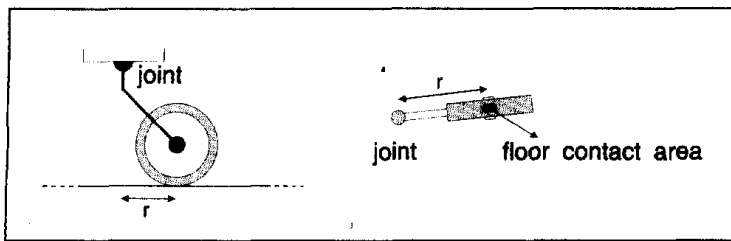

Fig. 4. Side-view and top-view of castor

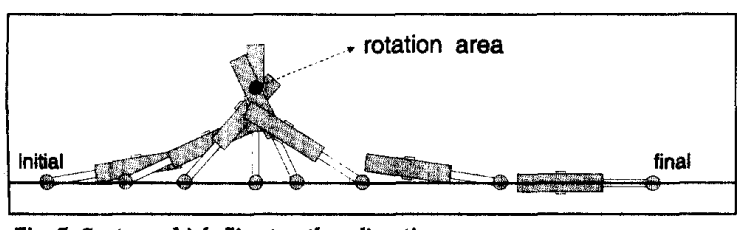

Fig. 5. Castor, which flips to other direction approximately $400 \mathrm{~kg}$. The length of the vehicle is $1.4 \mathrm{~m}$, its width is $1.0 \mathrm{~m}$ and its height is $0.8 \mathrm{~m}$.

The mechanical structure of the mobile robot has many properties which are not represented in the kinematic model (Eq. 1), the most prominent of which are Coulomb friction and compliance. The friction, which exists both in the driven wheels and the castor, is not constant, but depends strongly on the floor surface. The dominant compliance of the mechanical structure is due to the suspension, but also compliance of the transmission belts connecting the motors to the wheels cannot be neglected. These two main sources of compliance introduce resonance frequencies at $3 \mathrm{~Hz}$ and at 20 $\mathrm{Hz}$ in the controlled mobile robot. Another important property of the mechanical structure is its large inertia (almost 500 $\mathrm{kg}$ ), which causes large centripetal forces as the mobile robot rotates at high speed.

Coulomb friction in the driven wheels and their driving system introduces two types of errors. The total Coulomb friction introduces errors in driving direction. If the Coulomb friction differs at the left and right wheels, then there also is a disturbance torque acting on the vehicle, which causes orientation errors.

The effect of castor friction strongly depends on the orientation of the castor, relative to the vehicle. There are also two types of castor friction: rolling friction and rotational friction. The effect of the first can be compared to the friction effect of the driven wheels. It introduces a force opposite to the direction of rolling of the castor wheel. The latter type of friction is important if the castor-wheel has to rotate on the floor around a vertical axis, which passes through the center of the floor contact area (see also Figure 5).

In Figure 4 a side-view and a top-view of a castor are shown. The distance between the center of the floor contact area and the joint along the floor is denoted $k$; the symbol $r$.

In Figure 5 a top-view of a castor is shown whose joint is moving along a straight line. Initially the castor is pointing in the wrong direction, hence it flips to the other direction. The floor contact area moves along a curve with a cusp. At the cusp the castor-wheel rotates along a vertical axis, without rolling. After the cusp has been passed the rolling direction of the castor-wheel is reversed. Near the cusp, the effect of rotational friction is the strongest. The larger the floor contact area (softer tires have a larger floor contact area), the larger the rotational friction. This friction introduces a disturbance torque $T_{\mathrm{f}}$ at the wheel. This disturbance torque in turn induces a disturbance force $F_{\mathrm{j}}=T_{\mathrm{f}} / r$ at the castor joint. The distance $r$ between joint and floor contact area should hence be as large as possible. The force $F_{\mathrm{j}}$ causes the vehicle to move in a jerky way, when the castor flips from one direction to another. This effect is an important source of errors in mobile robot control if the vehicle has to move forward and backward.

The path followed by the castor as it flips from one direction to the other does not depend on the speed of the vehicle. This implies that the faster the castor-joint moves, the shorter the period during which the disturbance force $F_{\mathrm{j}}$ is felt. As the torque $T_{\mathrm{f}}$ and hence the force $F_{\mathrm{j}}$ are relatively independent of the velocity of the castor-joint (Coulomb friction), the integral $[F \mathrm{~d} d t$ becomes smaller when the castor-joint moves faster. This integral is a measure of the total disturbance of the mobile robot. Hence it is advantageous to move quickly while 
the castor flips to another direction.

The diverse friction components have been measured under varying conditions [5]. The average of a few tens of measurements shows that the total rolling friction when moving along a straight line with properly aligned castor is on the order of magnitude of $100 \mathrm{~N}$, where approximately $25 \mathrm{~N}$ is caused by rolling friction in both driven wheels and approximately $30 \mathrm{~N}$ by rolling friction of the castor. The remaining friction is in the motors and transmission. The friction is not always distributed symmetrically between the left and right driven wheels.

If the castor wheel is oriented perpendicularly to the direction of motion of the joint, then the force felt at the joint is approximately $80 \mathrm{~N}$. This force is caused by rotating friction. If the castor wheel is oriented in parallel to the direction of motion of the joint then an average force of approximately 30 $\mathrm{N}$ is felt at the joint. This force is caused by rolling friction of the castor wheel. For intermediate angles there is a combination of rolling and rotating friction.

At each driven wheel a force of $350 \mathrm{~N}$ can be delivered by the motors and transmission system.

\section{Velocity Control}

The hybrid stabilizing controller is designed for a system with velocity inputs. The mobile robot, however, has two force inputs. This problem is overcome by applying velocity control to the mobile robot. This velocity controller uses a simple planar dynamical model for compensation of Coulomb friction in the driven wheels and for compensation of centripetal forces.

The friction compensation does not distinguish between wheel friction and friction in the motors and transmission. A feedforward force of $30 \mathrm{~N}$ is used for each driven wheel. The direction of this force depends on the rolling-direction of the wheel. The total compensation is well below the total rolling friction of $100 \mathrm{~N}$. Too strong a compensation would result in a limit cycle. In that case the vehicle would be oscillating around a certain average pose. Friction in the castor is not compensated. This cannot be done in the practical setup, because of the lack of a sensor which measures the castor angle.

The centripetal force compensation may be as large as 50 $\mathrm{N}$ per driven wheel, when the angular velocity of the vehicle is close to its specified maximum value of $1 \mathrm{rad} \mathrm{s} \mathrm{s}^{-1}$.

Compliance of the suspension is not taken into account in the compensation. The suspension introduces resonance frequencies on the order of $3 \mathrm{~Hz}$. The bandwidth of the reference pose signal should be much lower than $3 \mathrm{~Hz}$.

A linear feedback law is superimposed on the compensation. Some experiments have been performed with the velocity-controlled vehicle in order to get an idea of its performance.

In Figures 6 and 7 hoth the reference signals (dashed lines) and the measured actual signals are given. Both control inputs were applied simultaneously. The figures show that in spite of the compensation the tracking accuracy is only moderate.

Figure 6 shows that the actual linear velocity is always less in magnitude than the reference linear velocity. This is due to the friction in the vehicle. By increasing the strength of the friction compensation this type of error can be reduced, but the chance of limit cycling increases. Figure 7 shows that the actual value of the angular velocity also tends to be lower in

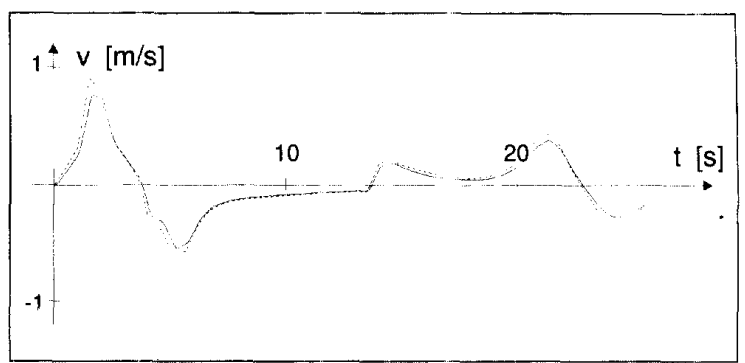

Fig. 6. Reference and measured linear velocity

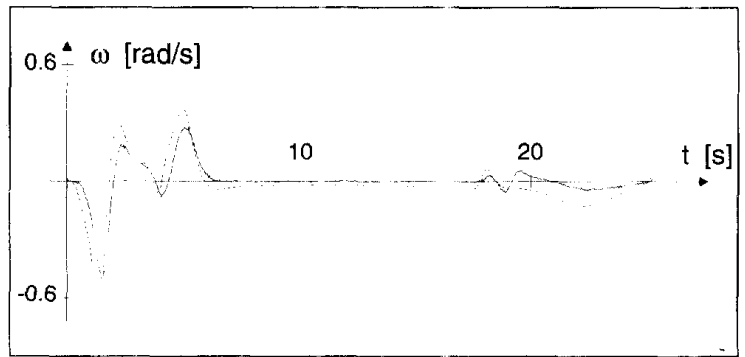

Fig. 7. Reference and measured angular velocity

magnitude than the reference value. Just before $t=20$ the castor flips from one orientation to the other. This results in a fast transition from negative to positive value of the angular velocity.

\section{Position Control}

The system, with velocity controller, can be regarded as a new system, with transfer $I+\Delta$ from $v_{\mathrm{d}}$ and $\omega_{\mathrm{d}}$ to $v$ and $\boldsymbol{\omega}$, where $\boldsymbol{I}$ is a unity transfer and where $\Delta$ is a perturbation term, due to the imperfect velocity controller. Specific $v(t)$ and $\omega(t)$ result in a certain $x(t), y(t)$ and $\varphi(t)$, determined by the kinematics of the vehicle. The hybrid controller can be built around the velocity-controlled system $I+\Delta$ (Figure 8 ).

Practical Setup of the Mobile Robot System The hybrid controller is implemented in OCCAM on a T800 transputer, running at $17.5 \mathrm{MHz}$. On the same transputer the velocity controller and some $1 / 0$ processes (file $\mathrm{I} / \mathrm{O}$, screen output and $\mathrm{AD} / \mathrm{DA}$ conversion) are running. The vehicle is connected to an 80386 host PC by means of a $10 \mathrm{Mbit} / \mathrm{s}$ transputer link. The controllers run at a sampling rate of $300 \mathrm{~Hz}$. This is well above the main resonance frequencies of the mechanical structure, which are $3 \mathrm{~Hz}$ and $20 \mathrm{~Hz}$.

The vehicle is placed in a room of approximately $6 \times 6 \mathrm{~m}^{2}$. This limits the allowable excursions of the vehicle to approximately $2 \mathrm{~m}$ around its initial position, which is in the middle of the room.

Measuring the position and orientation of the vehicle is done by integrating wheel revolutions (odometry).

\section{IMPLEMENTATION OF THE HYBRID CONTROLLER}

In the implementation of the hybrid controller a stabilizing linear feedback with constants $\alpha_{1}$ and $\alpha_{2}$ is chosen for K(.). A 


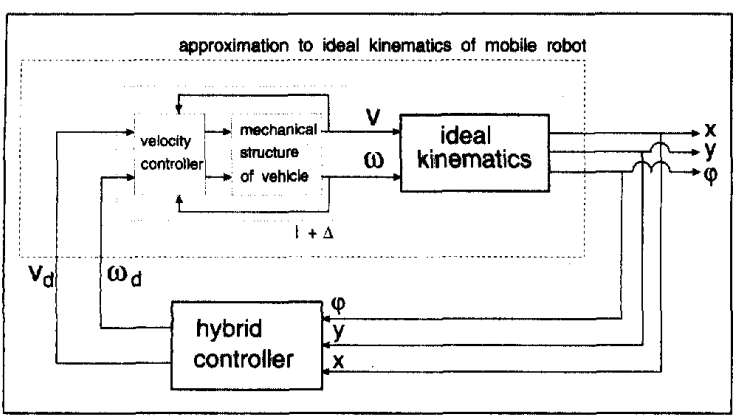

Fig. 8. Structure of position controlled system

quadratic function, multiplied by a constant $\alpha_{0}$, is chosen for $\alpha($.) (see also the previous discussion of the stabilization of extended one-chain systems):

$$
\begin{aligned}
& \mathbf{K}(\xi(k))=a_{1} x_{1}(k)+a_{2} w_{1}(k) \\
& \begin{aligned}
\alpha(r) & =\alpha_{0} r^{2} ; r^{2}=\|z(k)\|^{2} \\
& =w_{2}(k)^{2}+x_{2}(k)^{2}+x_{3}(k)^{2}
\end{aligned}
\end{aligned}
$$

Here a shorthand notation $\mathbf{z}(k)$ is introduced for $\mathbf{z}(k \delta)$. A similar notation is used for the elements of $\mathbf{z}(k \delta)$.

The error in driving direction is stabilized by the discrete time controller $\mathrm{K}($.) (Eq. 8a). The lateral error and orientation error are stabilized by the continuous-time controller (Eq. $8 \mathrm{~b})$. The second term of the discrete-time control signal, $v_{1}(k)$ (see also the previous discussion of the stabilization of extended one-chain systems), introduces a disturbance in the driving direction if there is a lateral error $\left(x_{3}\right)$, an orientation error $\left(x_{2}\right)$ or a curvature error $\left(w_{2}\right)$. The resulting controller has the following parameters:

$$
\begin{array}{ll}
\delta & \begin{array}{l}
\text { duration of the time interval during which } \\
v_{1}(t) \text { is constant } \\
\text { coefficients of characteristic polynomial of } \\
\text { lateral error dynamics } \\
k_{z j}(1 \leq j \leq 3) \\
a_{1} \text { and } a_{2}
\end{array} \\
a_{0} & \begin{array}{l}
\text { coefficients of discrete-time characteristic } \\
\text { polynomial of dynamics in the driving } \\
\text { direction } \\
\text { gain at which lateral and orientation errors } \\
\text { force the vehicle to move again in the } \\
\text { driving direction }
\end{array}
\end{array}
$$

The larger the parameter $\delta$, the longer the excursions made by the vehicle. The parameter $\delta$ was tuned such that the excursions fit safely in the available room. Parameters $k_{z_{j}}$ $(1 \leq j \leq 3)$ determine the three poles of the continuous-time subsystem with feedback. The suspension introduces a lowest resonance frequency of the velocity-controlled vehicle of approximately $3 \mathrm{~Hz}$, i.e. $20 \mathrm{rad} \mathrm{s}^{-1}$. The magnitude of the poles has to be much lower. They are chosen at -6 and $-2.5 \pm j 1.5$. Parameters $a_{1}$ and $a_{2}$, together with $\delta$, determine the two poles of the discrete-time subsystem with feedback $K($.). These poles are chosen such that the error in driving direction con- verges much slower than the lateral and orientation errors. Below follows a list of all parameter-values:

$$
\begin{aligned}
& \delta=0.5 ; \alpha=1 ; a_{1}=-0.36 ; a_{2}=-1.11 \\
& k_{z, 1}=11 ; k_{z, 2}=38.5 ; k_{z, 3}=51
\end{aligned}
$$

In the practical setup, the vehicle stops if the following margins are satisfied:

$$
\begin{aligned}
& |x|<0.01[\mathrm{~m}] \\
& |y|+c|\varphi|<0.10[\mathrm{~m}] \quad c=1\left[\mathrm{~m} \mathrm{rad}^{-1}\right] \\
& |v|<0.02\left[\mathrm{~m} \mathrm{~s}^{-1}\right] \\
& |\omega|<0.02\left[\mathrm{rad} \mathrm{s}^{-1}\right]
\end{aligned}
$$

These error margins define, as we previously stated, the neighborhood to which the system should converge for large $t$. Of course, given the model uncertainties, we have not exactly computed the design parameters $k_{z, j}(1 \leq j \leq 3), a_{1}, a_{2}, \alpha_{0}$ and $\delta$ as a function of the desired accuracy.

\section{EXPERIMENTAL RESULTS}

In the first experiments, a Euclidian norm was used for $\|z(k)\|$. In later experiments the norm was modified. This modification is based on insight in the physical meaning of $\|z(k)\|$ and the properties of the castor friction. This increased the performance of the controlled vehicle considerably.

\section{Experiments Using Euclidian Error-norm}

If there are only initial errors in driving direction and as long as the initial castor orientation is aligned properly with the vehicle's driving direction then the controller behaves well. Experiments with a non-zero initial lateral error show less satisfactory results. In most test runs the vehicle moved forward and backward without end. This is due to friction in the castor.

\section{Modification of Error-norm}

By retuning the parameters of section 4 , the behavior of the controlled system could be made slightly better, but no real improvements were obtained. The excitation in driving direction, due to lateral errors should be stronger, while the excitation, due to orientation errors and curvature errors should remain the same. This will be explained below. The excitation is introduced by means of the function $\mathrm{a}(\|z(k)\|)$, see $(8 \mathrm{a})$. In the initial setup a Euclidian norm was used for $\mid z(k) \|$, as was used by Canudas de Wit et al. [3] in their simulation studies:

$$
\|z(k)\|=\sqrt{w_{2}(k)^{2}+x_{2}(k)^{2}+x_{3}(k)^{2}}
$$

The coordinate $x_{3}(k)$ equals the lateral error $y(t)$ of the vehicle, at time $k \delta$. In order to selectively increase the coupling between lateral error and motion in driving direction, the error-norm $\|z(k)\|$ is redefined:

$$
\|z(k)\|_{\text {new }}=\sqrt{w_{2}(k)^{2}+x_{2}(k)^{2}+\alpha_{y} x_{3}(k)^{2}}
$$




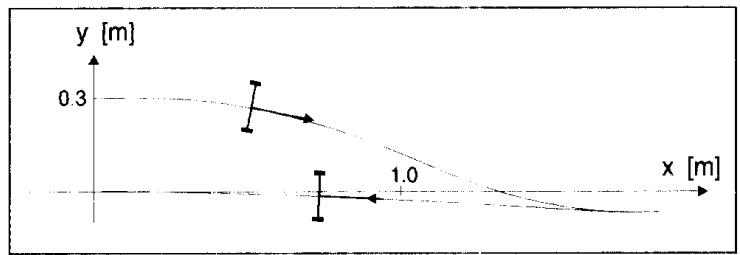

Fig. 9. Trajectory of vehicle in $x y$-plane

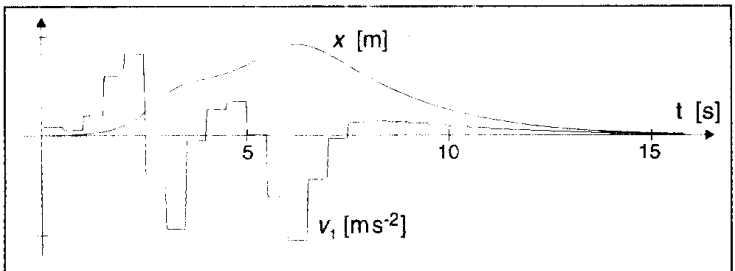

Fig. $10 v_{i}(k)$ and $x(t)$ as function of time

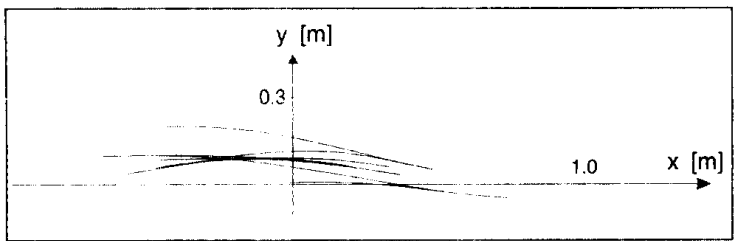

Fig. 11. Test run with initial errors $x=-0.5 \mathrm{~m}, y=0.2 \mathrm{~m}, \varphi=0 \mathrm{rad}$

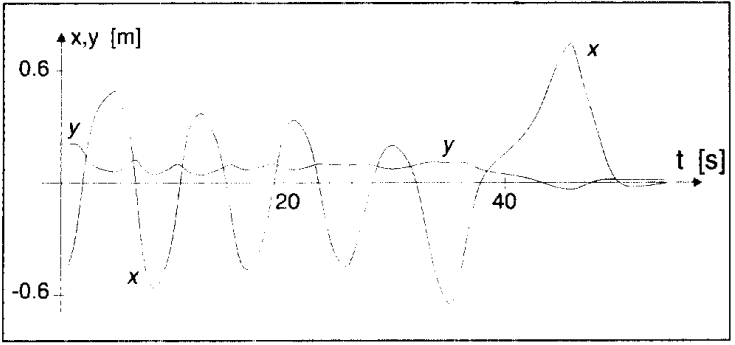

Fig. 12. Test run with $x, y$ as functions of time

A new parameter $\alpha_{y}$ is introduced, which is chosen equal to 3 . All other parameters remain the same. The norm given in (12) is equivalent to (13), with $\alpha_{y}=1$. The net effect of increasing $\alpha_{y}$ from 1 to 3 will be that the same lateral error makes the vehicle move more quickly in driving direction. This is advantageous, because at higher velocities in driving direction the castor needs less time to flip to the other direction. As the disturbance force hardly depends on the velocity, the total disturbance of the vehicle will be smaller (see also our previous discussion of castor friction). After this modification the vehicle reaches its end goal within a reasonable number of excursions.

Experiments with Modified Error-norm In Figure 9 a run is shown, with an initial lateral error of $0.3 \mathrm{~m}$. The covered trajectory is shown in the $x y$-plane.

Note the length of the excursion. It is approximately $1.8 \mathrm{~m}$ in the $x$-direction. After approximately 15 seconds the vehicle has reached its end goal, within a few centimeters. Another interesting feature is the control signal $v_{1}(\mathrm{k})$. Because of the initial non-zero lateral error the vehicle accelerates in the driving direction. Once the lateral error and orientation error are sufficiently compensated $v_{1}(k)$ converges to zero. Switching can be seen every half second. The control signal $v_{1}(k)$ is plotted in Figure 10, together with $x(t)$. The vertical axis ranges from $-2[\mathrm{~m}]$ to $+2[\mathrm{~m}]$ for $x(t)$ and from $-1\left[\mathrm{~ms}^{-2}\right]$ to $+1\left[\mathrm{~ms}^{-2}\right]$ for $v_{1}(k)$.

For compensation of the lateral error sufficiently long excursions should be made. This effect is demonstrated very well by another test run, see Figures 11 and 12. During this run the vehicle moves forward and backward several times. First, a number of excursions are made with a total length of less than $1 \mathrm{~m}$ in the driving direction. As soon as there is a longer excursion (total length approximately $1.5 \mathrm{~m}$ ), the lateral error can be compensated within the error margins. This behavior can be explained well by means of Figure 5. The castor needs a certain distance to flip from one direction to the opposite direction. When the excursion is short, then a relatively large part of the total excursion is used for changing direction of the castor. In that case the joint force is large during a large part of the excursion, which results in poor compensation of the lateral error.

Figure 11 shows the path of the mobile robot in the $x y$ plane and Figure 12 shows $x$ and $y$ as functions of time.

\section{CONCLUSIONS}

A hybrid controller [3], developed for non-holonomic systems, has been successfully implemented on the MART-robot, which is a two-degrees-of-freedom mobile robot with two driven wheels and a castor.

The controller excites the dynamics in driving direction as long as there is a non-zero lateral error, a non-zero orientation error or a non-zero curvature error of the mobile robot. This excitation causes the mobile robot to move forward and backward. Meanwhile the mobile robot can be steered towards a line where the lateral error equals zero. Castor friction causes large disturbance forces when the direction of motion of the mobile robot changes. This results in steering difficulties, especially when moving at low velocities. Insight into physical properties of the castor friction and a geometrical interpretation of the quantities appearing in the controller allowed us to increase the performance of the controller by selectively increasing the excitation in driving direction for lateral errors.

As the castor needs a certain distance to flip from one direction to the other, the controller performs better if longer excursions of the vehicle are allowed during stabilization. This can be achieved either by increasing the discrete sampling interval of the controller or by making the dynamics of the discrete-time part of the controlled system slower (i.e., making the dynamics in the driving direction slower). The use of slower dynamics in driving direction does not imply motion at lower velocities, but it implies a lower rate of change of the velocity.

The vehicle does not stop exactly at the desired goal point, but a certain error remains. This error could be explained by the castor friction, which prevents the vehicle from steering 
at very low speeds. However, the error could be attributed to a fundamental property of the hybrid controller itself: this is not an asymptotically stabilizing controller, see [3]. Only a certain upper error bound can be given for a set of controller parameters. It is expected that the castor friction increases the non-zero upper bound, which already exists for an ideal mobile robot. This subject, however, needs further investigation.

\section{REFERENCES}

[1] Abrahams J. (1985), Zeer voorlopig onderzoek naar flexibele assemblage systemen (in Dutch), CFT Philips, Eindhoven, The Netherlands.

[2] Brockett, R.W. (1983), Asymptotic stability and feedback linearization, Differential Geometric Control Theory, pp. 181-191, Birkhäuser.

[3] Canudas de Wit, C., H. Berghuis, and H. Nijmeijer (1994), Hybrid stabilization of Nonlinear Systems in Chained Form, Proc. IEEE Conf. on Decision and Control, pp. 692-697, Lake Buena Vista.

[4] Coron, H.-M., and J.-B. Pomet (1992), A remark on the design of time-varying stabilizing feedback laws for controllable systems without drift, Proc. IFAC Symp. Nonlinear Control Systems Design, pp. 413-417, Bordeaux, France.

[5] Idema L.J. (1992), Onderzoek naar de invloed van wrijving op het rijgedrag van een mobiele robot (in Dutch). University of Twente, Dept. of Electrical Engineering, Control Laboratory, report nr. 92R115.

[6] Murray, R.M., and S.S. Sastry (1993), Nonholonomic Motion Planning: Steering Using Sinusoids, IEEE Trans. Automatic Control, vol. 38 , pp. $700-716$

[7] Murray, R.M., and S.S. Sastry (1991), Steering non-holonomic systems in chained form, Proc. IEEE Conf, on Decision and Control, pp. 1121-1126, Tucson, Arizona.

[8] Oelen, W., H. Berghuis, H. Nijmeijer and C. Canudas de Wit (1994), Implementation of a Hybrid Stabilizing Controller on a Mobile Robot with Two Degrees Of Freedom, IEEE Conf. on Robotics and Automation, San Diego, pp. 1196-1201, San Diego, California

[9] Pomet, 3.-B. (1992), Explicit design of time-varying stabilizing control laws for a class of controllable systems without drift, Systems and Control Letters, vol. 18, pp. 147-158.

[10] Samson, C. (1990), Velocity and torque feedback control of a nonholonomic cart, Int. Workshop on Adaptive and Nonlinear Control: Issues in Robotics, Grenoble, France, Nov. 21-23, 1990, pp. 125-151. Proc. in Advanced Robot Control, Vol. 162, Springer Verlag, C. Canudas de Wit (Ed).

[11] Sørdalen, O.J. (1993), Canversion of the kinematics of a car with $n$ trailers into a chained form, IEEE Conf. on Robotics and Automation, Atlanta, Georgia.

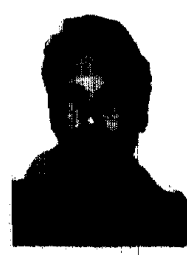

Carlos Canudas de Wit received his B.Sc. degree in electronics and communications from the Technologic of Monterey, Mexico, in 1980. From 1981 to 1982 he worked as a research engineer in the Department of Electrical Engineering at the CINVESTAVIPN in Mexico City. In 1984 he received his M.Sc. from the Polytechnic of Grenoble (Department of Automatic Control), Grenoble, France. He was a visiting researcher in 1985 at Lund Institute of Technology, Sweden. In 1987 he received his Ph.D. in automatic control from the Polytechnic of Grenoble (Dept. of Automatic Control), France. Since that time he has been an associate professor in the Department of Automatic Control, Polytechnic of Grenoble, where he teaches and conducts research in the area of adaptive and robot control. In 1988 he authored Adaptive Control of Partially Known Systems: Theory and Applications (Elsevier). He is also editor of Advanced Robot Control (Springer-Verlag, 1991). He is currently an associate editor of the IEEE Transactions on Automatic Control.

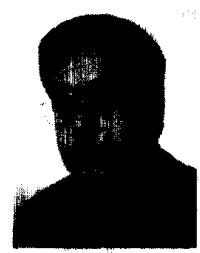

Henk Nijmeijer (M'83-SM'91) received both the university degree and the doctorate in Mathematics from the University of Groningen, The Netherlands, in 1979 and 1983 , respectively. After spending three years at the Centre for Mathematics and Computer Science (CWI) in Amsterdam he moved to the Department of Applied Mathematics of the Twente University of Technology in 1983, where he is presently an associate professor (UHD). His main research interests are in the area of nonlinear and linear control theory and its applications. He is the author (with A.J. van der Schaft) of the book Nonlinear Dynamical Control Systems (Springer, 1990).

Dr. Nijmeijer is a member of the editorial board of the SIAM Joumal on Control and Optimisation, the Intermational Journal of Robust and Nonlinear Control, the Journal of Dynamical and Control Systems and the IFAC Journal Automatica.

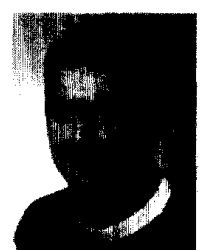

Harry Berghuis was born on September 14, 1965, in Westerhaar, The Netherlands. He received a M.Sc. degree in Electrical Engineering from the University of Twente, Enschede, The Netherlands. in 1988 where he also completed his Ph.D. research on model-based robot control. Since 1993 he has been with the Control Group at Hollandse Signaalapparaten B.V. in llengelo, The Netherlands, where he develops radar control systems.

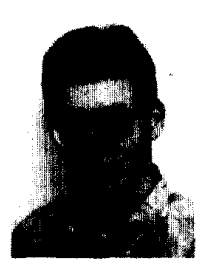

Wilco Oelen was born January 25, 1966, in Hoogeveen, the Netherlands. In 1990 he completed the M.Sci in Electrical Engineering at the University of Twente, specializing in computer science. His master's thesis was on the use of neural networks in control engineering. Immediately after finishing his studies, Mr. Oelen joined the Department of Computer Sciences, also at the University of Twente, working on a real-time operating system, running on a network of transputers. From 1990 to 1994 he worked on his Ph.D. studies on the design process of mechatronic systems. Important aspects of mechatronic design are the use of modeling tools and the use of control theory. Mr. Oelen expects to finish his Ph.D. studies in June 1995. Since November 1994 he has been with the Computer Management Group (CMG) in Amstelveen since the first of November 1994. where he is working in the area of telecommunications and client/server systems, based on TCP/IP networking. 